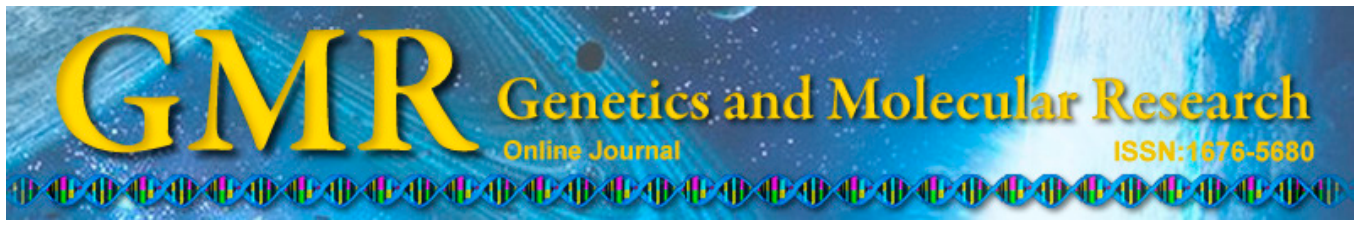

\title{
Treatment and mechanism of BMMSCs on deep II degree scald of hamster skin
}

\author{
M. Ma ${ }^{1 *}$, T. Jiang ${ }^{2 *}$, N. Li ${ }^{1}$, A. Aliya ${ }^{1}$ and A. Tuhan ${ }^{1}$ \\ ${ }^{1}$ The Teaching Management Department, \\ The First Affiliated Hospital of Xinjiang Medical University, \\ Urumqi, Xinjiang, China \\ ${ }^{2}$ Clinical Research Institute of Xinjiang Medical University, Xinjiang, China \\ *These authors contributed equally to this study. \\ Corresponding author: A. Tuhan \\ E-mail: 315012896@qq.com
}

Genet. Mol. Res. 14 (3): 8244-8251 (2015)

Received September 29, 2014

Accepted February 19, 2015

Published July 27, 2015

DOI http://dx.doi.org/10.4238/2015.July.27.12

\begin{abstract}
In this study, we examined the treatment and mechanism of BMMSC on a deep II degree scald of the hamster skin. A deep II degree scald model on the skin of 40 hamsters was duplicated and divided randomly into a stem cell plantation group (group A) and model control group (group B). Skin cells were cultured in vitro until the allogeneic BMMSCs of the 5 th generation formed with a cell count of $1 \times 10^{7} / \mathrm{mL}$. Local injection plus liquid supernatant smearing was used to plant the cells into the position of the scald in the stem cell plantation group. The control group was given an equivalent amount of normal saline to observe the healing action, and 5 samples were taken in each group after 1, 3, 7, and 14 days for hematoxylin and eosin staining for physiological observation. Polymerase chain reaction was used to detect the amount of chymotrypsin in mast cells. The speed of healing in the stem cell transplantation group was greater than that in the control group; staining results showed that the quality of healing in the transplantation group was better than that in the control group. Chymotrypsin expression was detected in both groups, reaching a peak
\end{abstract}


on day 3. BMMSCs can accelerate wound healing, and chymotrypsin in mast cells may participate in the wound healing process.

Key words: Bone marrow mesenchymal stem cells; Chymotrypsin; Hamster; Scald

\section{INTRODUCTION}

Bone marrow mesenchymal stem cells (BMMSCs) exhibit advantages such as easy acquisition, are free from rejection, and can rehabilitate and substitute for damaged tissues. Although it has been reported that BMMSCs can accelerate the healing of wound of skin (Liu, et al., 2007), the molecular biological mechanism remains unknown. Recent domestic and foreign studies showed that the medium and cytokine the skin mast cells released can activate inflammatory cells in the vascular endothelial nucleus to defend against inflammation (Marshall and Jawdat, 2004). Moreover, chymotrypsin can facilitate the transformation of angiotensin (Ang I) to Ang II in the damaged tissue and promote the synthesis of fibrocytes and expression of transforming growth factor (Reid et al., 2007). Therefore, understanding the relationship between BMMSCs and wound healing as well as chymotrypsin expression in mast cells would be helpful for determining the mechanism of BMMSCs in repairing wounded tissue.

\section{MATERIAL AND METHODS}

\section{Experimental animals}

Forty hamsters aged 8 weeks were purchased from Xinjiang Experimental Animals Center. The entire experiment was completed in the Experimental Animals Science Department in the First Teaching Hospital of Xinjiang Medical University. The experimental protocol was reviewed and approved by the Usage and Management Committee of Animals of the First Teaching Hospital of Xinjiang Medical University, with the ethic lot number A-20100917001. The experiments were conducted under the supervision of the organization.

\section{Main reagents}

L-DMEM culture medium was obtained from Gibco (Grand Island, NY, USA). Fetal calf serum was from Hangzhou Sijiqing Biological Materials Co., Ltd. (Hangzhou, China). Trizol was from Invitrogen (Carlsbad, CA, USA). SYBR Premix Ex Taq was from Takara (Shiga, Japan). Primers were synthesized by Shanghai Shengon Biotech Engineering Co., Ltd. (Shanghai, China).

\section{Duplication of model and grouping in the experiment}

\section{Isolated culture and identification of BMMSCs}

Four male hamsters aged 8 weeks were euthanized by cervical dislocation and sterilized using iodophor and ethyl alcohol. The femur and tibia were removed and placed in culture medium under aseptic conditions and then immersed in L-DHANKS solution containing 200 $\mathrm{U}$ penicillin and streptomycin for $10 \mathrm{~min}$. The samples were removed and rinsed twice with 
D-HANKS. Next, the 2 epiphyseal ends were sheared and the marrow cavity was washed repeatedly with L-DMEM until the marrow cavity became white. Samples were centrifuged for $5 \mathrm{~min}$ at $10,000 \mathrm{~g}$ at $4^{\circ} \mathrm{C}$. Marrow cells were rinsed twice with L-DMEM solution and 2 drops were removed from the mixture and suspended in marrow cell solution. Trypan Blue staining and counting were made. Cell density was adjusted to $1 \times 10^{7} / \mathrm{mL}$ and add the culture liquid (15\% fatal calf serum). Cells inoculated into $25-\mathrm{cm}^{2}$ culture bottles were placed in the culture box at $37^{\circ} \mathrm{C}$ and $5 \% \mathrm{CO}_{2}$ under saturated humidity. The solution was first exchanged at $72 \mathrm{~h}$, and then every 3 days. Cells were collected at the 5 th generation when the solution was exchanged (P5). Flow cytometry was used to detect the expression of the CD29, CD44, and CD105 markers on the cell surface to identify the cells.

\section{Preparation of deep II degree scald model}

Ketamine hydrochloride ( $45 \mathrm{mg} / \mathrm{kg}$ body mass) was used to anesthetize the animals in the abdominal cavity. Next, sodium sulfide depilating agent was applied to the back to prepare the skin. The temperature of the thermostatic water bath was adjusted to $75^{\circ} \mathrm{C}$. A $50-\mathrm{mL}$ injector with a diameter of $3 \mathrm{~cm}$ was placed in the back of the animal, and $20 \mathrm{~mL}$ water at $75^{\circ} \mathrm{C}$ was injected for $12 \mathrm{~s}$ to prepare the deep II degree scald model, which was verified by physiological cutting.

\section{Grouping of animals}

The scald models prepared were randomly divided into a stem cell transplantation group (group A) and model control group (group B) including 20 animals in each group. The stem cell group was treated with the in vitro BMMSC culture, using $1 \times 10^{7} / \mathrm{mL} 5$ th generation cells immediately after scalding. Five spots were chosen in the scald location and each spot was locally injected with $0.1 \mathrm{~mL}$ stem cells; $0.5 \mathrm{~mL}$ liquid supernatant on a sterile cotton swab was smeared on the scalded location. The wound was then covered with sterile gauze. Attention was paid to the heat preservation of the animals. The control group was given the equivalent amount of normal saline using the same method as in the model group. The animals were raised separately in the small cages.

\section{Observation of indicators}

The animals were raised in separate cages with food and water ad libitum. Each day, the animals were observed to determine mental state, activity levels, and granulation tissue growth and wound healing. Five animals were euthanized on days 1, 3, 7, and 14 after transplantation and their materials were collected. The wound skin was cut into regular slices and hematoxylin and eosin staining and microscopy were conducted.

\section{Detection of activity and quantity of chymotrypsin}

Wound tissues at different time points were analyzed by real-time fluorescent quantitative polymerase chain reaction (PCR) to determine the expression level of chymotrypsin. A genic primer for chymotrypsin was synthesized by Takara. The upstream primer sequence was 5'-CTG AGA GGA TGC TTC TT CTG C-3', while the downstream primer sequence was 5'-AGA TCT TAT TGA TCC AGGCC G-3'; $\beta$-actin: upstream primer: 5'-AAC TCC ATC ATG AAG TGTGA-3', downstream primer: 5'-ACT CCT GCT TGC TGA TCC AC-3'. 
RNA was extracted using Trizol, chloroform, isopropanol, 75\% alcohol and diethylpyrocarbonate, then stored at $-80^{\circ} \mathrm{C}$. Next, $1.5 \mu \mathrm{g}$ extracted RNA was mixed with $1 \mu \mathrm{g}$ oligo (dT) primer, $1 \mu \mathrm{L}$ reverse transcriptase $4 \mu \mathrm{L} 2 \mathrm{X}$ first-strand buffer, and water for a total volume of $20 \mu \mathrm{L}$. After centrifugation, the sample was placed at $37^{\circ} \mathrm{C}$ for $60 \mathrm{~min}$ and then at $85^{\circ} \mathrm{C}$ for $5 \mathrm{~min}$. The sample was then cooled on ice. The sample was centrifuged at high speed at room temperature for $5 \mathrm{~s}$. All the supernatant was collected and reserved under $-20^{\circ} \mathrm{C}$.

The fluorescence quantitative PCR reaction system contained the following: 10 L SYBR Premix Ex Taq, 0.25 L 50 M upstream primer, 0.25 L $50 \mathrm{M}$ downstream primer, $4.5 \mathrm{~L}$ sterile deionized water, and $5 \mathrm{~L}$ reverse-transcribed cDNA, for a total volume reaction volume of $20 \mathrm{~L}$.

The amplification conditions were as follow: 1) $95^{\circ} \mathrm{C}$ pre-denaturation for $3 \mathrm{~min}$; 2) $95^{\circ} \mathrm{C}$ denaturation for $45 \mathrm{~s}, 55^{\circ} \mathrm{C}$ annealing for $45 \mathrm{~s}, 72^{\circ} \mathrm{C}$ extension for $45 \mathrm{~s}$, for 35 cycles, with fluorescence detected after each cycle and extension, and the analysis mode was set as quantitative analysis; 3) $72^{\circ} \mathrm{C}$ extension for $5 \mathrm{~min}$. For $\beta$-actin, amplification conditions were: 1) $95^{\circ} \mathrm{C}$ pre-denaturation for $\left.4 \mathrm{~min} ; 2\right) 95^{\circ} \mathrm{C}$ denaturation for $30 \mathrm{~s}, 60^{\circ} \mathrm{C}$ annealing for $30 \mathrm{~s}$, and $72^{\circ} \mathrm{C}$ extension for $45 \mathrm{~s}$ for 35 cycles with fluorescence detected after each cycle and extension and the analysis mode was set as quantitative analysis; 3) $72^{\circ} \mathrm{C}$ extension for $2 \mathrm{~min}$. The final temperature set was $72^{\circ} \mathrm{C} 7 \mathrm{~min}$ (temperature change rate $0.1^{\circ} \mathrm{C} / \mathrm{s}$ ). Fluorescence was monitored continuously, and dissociation curve analysis was conducted. The instrument detection channel was F1 channel.

\section{Statistical analysis}

The data statistically analyzed using the software SPSS17.0 (SPSS, Inc., Chicago, IL, USA). Measurement data are reported as means \pm SE. The comparison between two groups was detected for the 2 separate samples. The difference was considered to be statistically significant when $\mathrm{P}<0.05$.

\section{RESULTS}

\section{MSC results}

The positive rates of MSC specific surface antigen CD29 and CD44 were 98.8 and $96.6 \%$, and CD29 and CD44 showed positive expression in flow cytometry analysis (Figure 1).
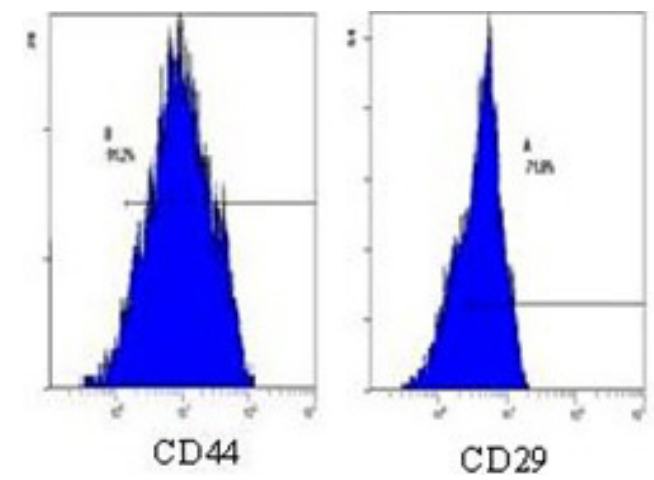

Figure 1. Testing result of CD29 and CD44. 


\section{Skin histopathology of hamster with deep II degree scald}

Histopathological detection showed that burning for $12 \mathrm{~s}$ caused hamster skin deep II degree scalding and damage to the deep dermis (Figure 2).

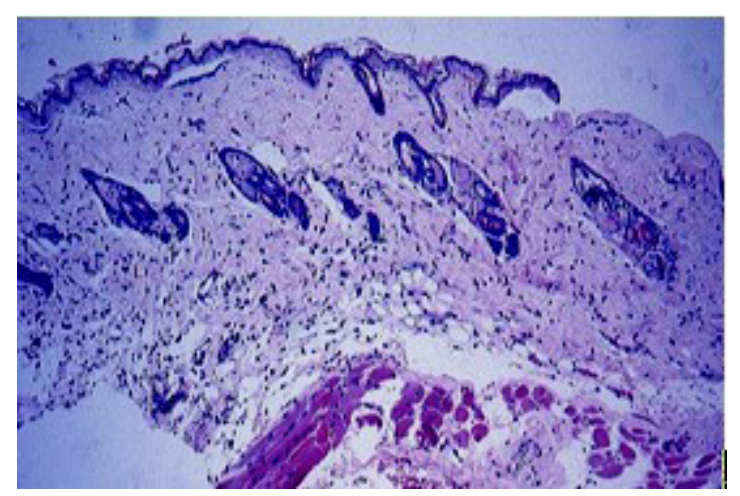

Figure 2. $\mathrm{H} \& \mathrm{E}$ staining of wound skin tissues at $75^{\circ} \mathrm{C}, 12 \mathrm{~s}(\mathrm{H} \& \mathrm{E}, 100 \mathrm{X})$.

\section{Wound healing in deep II degree scald model}

The skin of the animals in the 2 groups became red and swollen immediately after deep II degree scalding. Six hours later, the surface had turned gray. Twenty-four hours after scalding, the surface began to harden. After $72 \mathrm{~h}$, the skin in the stem cell transplantation group began forming an eschar. The difference in scabbing time for groups A and group B was not remarkable $(\mathrm{P}>0.05)$. For healing rate, $90 \%$ was considered to be the reference standard. Fourteen days after scalding, the difference between group A and group B was significant ( $\mathrm{P}$ $<0.05)$ (Table 1).

Table 1. Ratio of wound healing of all groups at different times (\%).
\begin{tabular}{llccc}
\hline Groups & \multicolumn{4}{c}{ Time (days) } \\
\cline { 2 - 5 } & 1 & 3 & 7 & 14 \\
\hline Group A & 0 & $3.38 \pm 2.35$ & $24.38 \pm 4.89$ & $54.78 \pm 2.83^{*}$ \\
Group B & 0 & $3.15 \pm 2.46$ & $21.34 \pm 3.26$ & $45.36 \pm 3.47$ \\
\hline
\end{tabular}

Compared with the group $\mathrm{B}, * \mathrm{P}<0.05$.

\section{Tissue morphology in deep II degree scald model}

Seven days after scalding, observation of fibro cells in group A under the microscope showed that number of blood capillaries was much richer than those in group B, and small amount of collagen tissue had formed. At 14 days, compared the control group, the blood capillary tissue increased remarkably and a granulation tissue layer had formed, and a large number of collagen settled (Figure 3). 


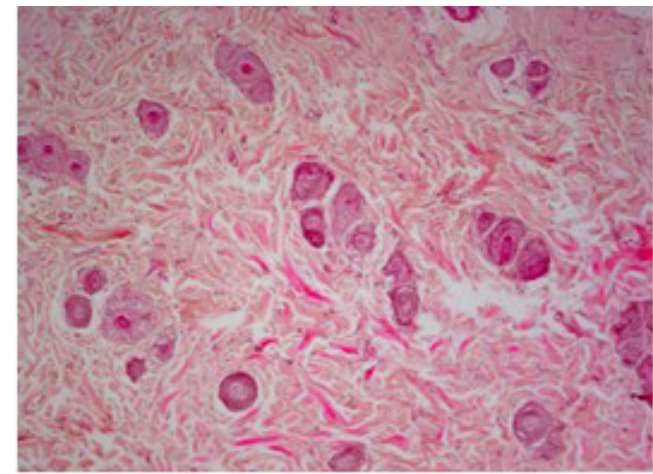

Group A

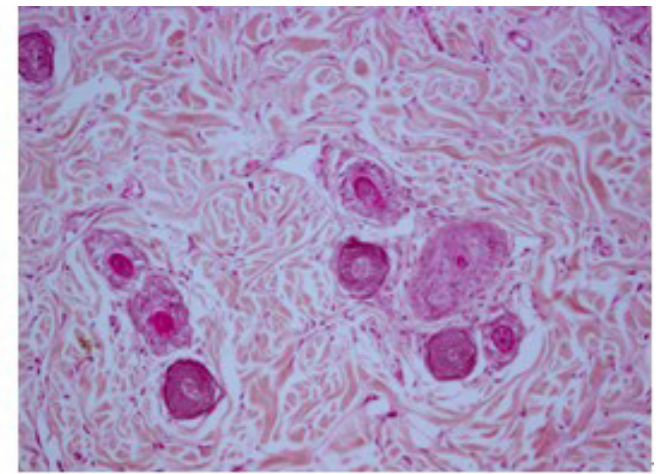

Group B

Figure 3. H\&E staining of wound skin tissues of the 14th day (H\&E, 200X).

\section{Expression of chymotrypsin genes in deep II degree scald model}

Chymotrypsin genes were detected in wound tissues at $1,3,7$, and 14 days after scalding using fluorescent quantitative PCR; chymotrypsin was observed at all of the time points. The difference in the 2 groups in the detection of genes was not significant $(\mathrm{P}>0.05)$. However, compared with the level of chymotrypsin mRNA at each time point, the highest expression occurred on the 3rd day. Compared with the control group, the level of mRNA days 7 and 14 in the transplantation group showed a significant difference $(\mathrm{P}<0.05)$ (Figures 4 and 5).

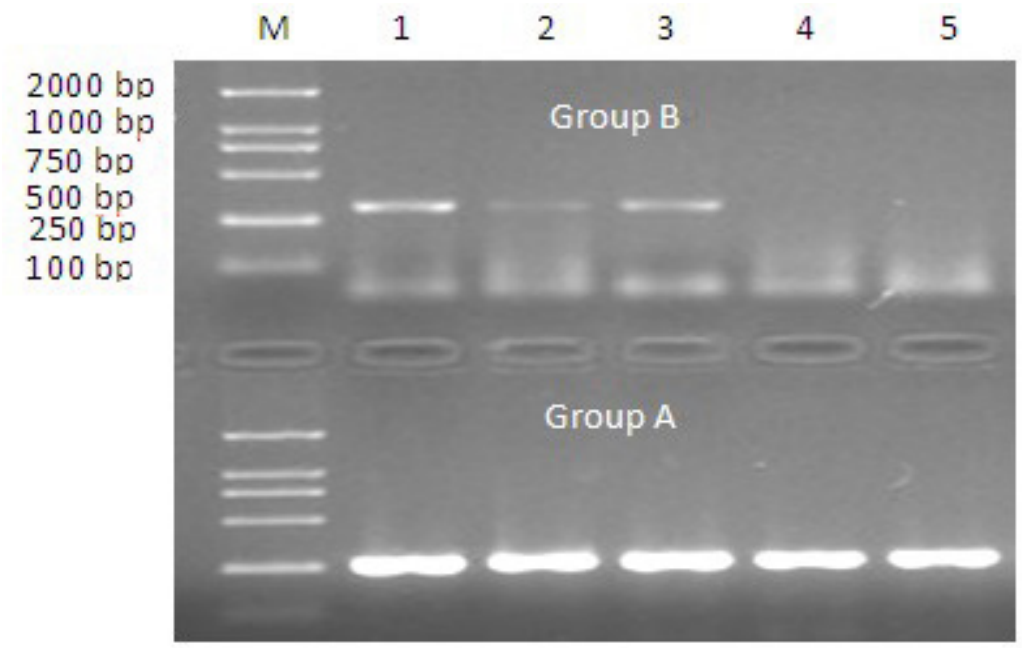

Figure 4. RT-PCR analysis of expressions of chymase in scald wound. Lane $M=$ marker, lane $1=$ control; lanes 2, $3,4,5=1,3,7$, and 14 days, respectively. 


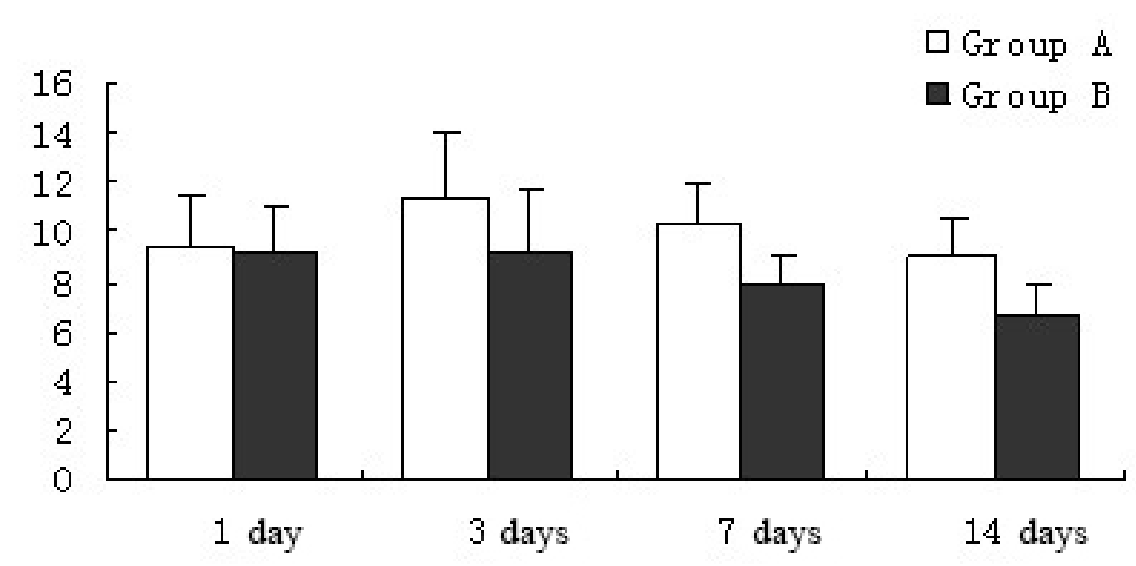

Figure 5. Chymase mRNA levels at different time periods. Compared with the group $\mathrm{B}, * \mathrm{P}<0.05$.

\section{DISCUSSION}

In recent years, the action of MSCs in wound rehabilitation has been examined in many studies. The source of marrow was examined, and the potential for multiple differentiation and easy isolation and culture were determined. Additionally, these cells do not differentiate automatically during culture and can be induced into diverse cell types. This provides a new treatment method for repairing damaged or pathological tissues in humans. Liu et al. (2006) suggested that BMMSCs expressed the epidermal growth factor, vascular endothelial growth factor, and fibroblast growth factor. These cytokine plays an important role in adjusting and creating the cell phenotype. Multiple cell growth factors mediate and regulate fibrosis, vascularization, and re-epithelialization at wound sites in the skin to promote wound healing (Ding and Tang, 2000). Nakagawa et al. (2005) suggested that a combination of MSCs and alkaline fibroblast growth factor can accelerate skin healing.

Additionally, the regeneration and repair course during the inflammation reaction participating in wound healing was revealed. The wound led to damage to endothelial cells, as well as blood platelet gathering and fibrin clot formation. Blood platelets released fibroblast growth factor, platelet derived growth factor, transforming growth factor, epidermal growth factor, and vascular endothelial growth factor. The inflammatory reaction has an important influence on the course wound rehabilitation. Mast cells in the skin cannot only release amine and fatty medium in the form of degranulation, but also release a large amount of cytokine and chemotactic factor (Kobayashi et al., 2000). Some studies showed that chymotrypsin in mast cells can complete the transformation from Ang I to Ang II. The results of the present studies showed that chymotrypsin can be expressed at different time points and to a higher degree on the 3rd day after a wound, which agrees with the results of Dong et al. (2011). Compared with the control group, the number of fibroblasts and blood capillary tissue in the transplantation group increased significantly on days 7 and 14, and the expression of chymotrypsin was higher than in the control group. Thus, BMMSCs may have accelerated the expression of chymotrypsin in mast cells and chymotrypsin in mast cells promoted the differentiation of fibroblast cells to promote wound healing. 
In summary, wound rehabilitation is an extremely complicated histological, biologi$\mathrm{cal}$, and chemical process. The action of chymotrypsin and its molecular mechanism require further analysis.

\section{Conflicts of interest}

The authors declare no conflict of interest.

\section{ACKNOWLEDGMENTS}

Research supported by grant (PT1205) from Condition Platform Construction Project of The Xinjiang Uygur Autonomous Region Science and Technology Department.

\section{REFERENCES}

Ding R and Tang X (2000). Advances in cell biology of wound healing. J. Trauma Surg. 2: 59.

Dong X-L, Geng Z-L, Gao W-C, Zhao Y, et al. (2011). Chymase expression in deep partial-thickness burn wounds after burn injury in hamsters. J. Clin. Rehabilitative Tissue Eng. Res. 15: 8681-8684.

Liu Y, Dulchavsky DS, Gao X, Kwon D, et al. (2006). Wound repair by bone marrow stomal cells though growth factor production. J. Surg. Res. 136: 336-341.

Liu P, Liu T, Li Y, Deng ZH, et al. (2007). Bone marrow-derived mesenchymal stem cells in the acceleration of skin wound healing. J. Fourth Mil. Med. Univ. 28: 1208-1210.

Kobayashi H, Ishizuka T and Okayama Y (2000). Human mast cells and basophils as sources of cytokines. Clin. Exp. Allergy 30: 1205-1212.

Marshall JS and Jawdat DM (2004). Mast cells in innate immunity. J. Allergy Clin. Immunol. 114: 21-27.

Nakagawa H, Akita S, Fukui M, Fujii T, et al. (2005). Human mesenchymal stem cells successfully improve skin-substitute wound healing. Br. J. Dermatol. 153: 29-36.

Reid AC, Silver RB and Levi R (2007). Renin: at the heart of the mast cell. Immunol. Rev. 217: 123-140. 